dessen Präparate erhebliche Mengen Cinchonidinsulfat enthielten und daher 14,38 - 14,80 Proc. Krystallwasser enthalten konnten, ohne verwittert zu sein. Die Kerner'sche Probe würde allerdings diesen Irrthum zur Genüge entschuldigen.

Es mag hier beigefiugt werden, dass ich wiederholt im Handel ein Chininsulfat antraf, welches stark verwittert war und deshalb nur 10-11 Proc. Krystallwasser enthielt. Dieser geringere Krystallwassergehalt gab mir stets Veranlassung, das fragliche Sulfat auf Cinchonidin zu prüfen und dasselbe eventuell zu bestimmen. Dabei wurden nun Resultate erhalten, aus welchen sich berechnen liess, dass der höhere Handelswerth, den das anscheinend gute Sulfat besass, indem es nur 10-11 Proc. Krytallwasser enthielt, auf der andern Seite durch den Gehalt an Cinchonidinsulfat reichlich wieder ausgeglichen wurde.

\title{
Chinologische Bemerkungen.
}

\author{
Von Demselben.
}

Unter dem Titel „zur Geschichte des Chinidins und Cinchonidins und Normirung der Nomenclatur dieser Chinaalkaloïde durch den chinologischen Congress in Amsterdam (13. April 1877)" veröffentlicht Herr Kerner in diesem Archiv, 216, 259, eine Mittheilung, in welcher die Geschichte dieser Alkaloïde einer argen Misshandlung unterworfen wurde. $\mathrm{Da}$ sich noch andere Ungenauigkeiten in jener Mittheilung vorfinden und dieselbe, im Ganzen genommen, gegen mich gerichtet ist, so habe ich mich dadurch veranlasst gesehen, einige Bemerkungen dazu zu geben.

Herr Kerner führt zunächst aus, dass bislang, namentlich früher, unter dem Namen Chinidin im Handel verschiedene Präparate verstanden wurden, was dadurch veranlasst worden sei, dass das Chinidinsulfat des Handels durch Zusammenkrystallisiren verschiedener Sulfate von Chinaalkaloïden erhalten wurde und nun diese Gemische bisweilen direct für besondere Substanzen angesehen worden seien. Er selber habe einen solchen Fehler begangen, indem er 1862 zwei solche gemischte Präparate als Modi ficationen des einen Alkaloïds ansab, das ich später aus diesem Wirrwarr ( $\alpha-, \beta-, \gamma$-Chinidin) herauszog und Conchinin nannte. 
In Betreff des $\alpha$-Chinidins lesen wir in jener Mittheilung (1862), dass dasselbe nichts anderes als das gewöhnliche Chinidin sei, dessen Sulfat namentlich vor jener Zeit im Handel vorkam. Herr Kerner ist dann später (1869) nochmals auf dieses Alkaloïd zurückgekommen, nannte es aber nun kurz Chinidin, während ihm für sein $\beta$-Chinidin die von mir gewählte Bezeichnung Conchinin passend erschien, wie weiter unten gezeigt wird.

Ich habe dann 1873 in eine Abhandlung, betitelt "Studien über die Alkaloïde der Chinarinden ", dieses $\alpha$-Chinidin oder damals schon kurzweg Chinidin genannte Alkaloüd mit aufgenommen, jedoch meive Zweifel über die Individualität dieser Base ausgesprochen. In Folge dessen schrieb mir Herr Kerner am 11. Sept. 1873 wie folgt: „Obschon ich noch heute fest von der Existenz des seiner Zeit als $\alpha$-Chinidin beschriebenen Chinidins, wie es vor 12 - 15 Jahren namentlich im englischen Handel vorkam, überzengt bin, so konnte ich es doch seither nicht mehr in dem Chinidin des Handels und in keiner Rinde auffinden."

Aus der neulichen Mittheilung erfahren wir nun, dass dieses $\alpha$-Chinidin wahrscheinlich ein mit Cinchonidin verunreinigtes Conchinin gewesen ist, also eine oberflächlich untersuchte Substanz. Gleichwohl hielt Herr Kerner die Individualität dieser Substanz nicht nur bis zum Jahre 1869 aufrecht, sondern bezeichnete auch in jenem Jahre verschiedene Substanzen, welche ein Gemisch von diesem Chinidin mit andern Alkaloïden sein sollten. So giebt derselbe z. B. an, dass or im Handel ein Aricinsulfat antraf, dessen basischer Antheil aus Chinidin und Cinchonidin bestehen solle.

Später übersandte mir Herr Kerner das fragliche Aricinsulfat und versetzte mich somit in die Lage, dieses Chinidin näher ansehen zu können. Hierbei fand ich nun, dass das fragliche Aricin ausser Cinchonin, seinem Cinchonidin (d. i. Homocinchonidin) nur Winckler's Chinidin enthielt, woraus ich nun schloss, dass jenes $\alpha$-Chinidin oder gewöhnliche Chinidin eben nichts anders sei als dasjenige Alkaloïd, welches ich gegenwärtig Cinchonidin nenne. Mit dieser Auffassung stimmt auch vollkommen das Resultat der Untersuchung von Chinidin. purum und Chinidin. sulfuricum, welche Präparate Herr Kerner 1858 dem Apotheker Reichel in Dresden gab und die sich gegenwärtig in meinem Besitze befinden.

Wenn daher Herr Kerner neulich behauptete, ich hätte Schrage eine grundfalsche Belehrung in Sachen des Chinidins zukom- 
men lassen, so fällt dieser Tadel auf Herr Kerner zurück, da derselbe eine grundfalsche Untersuchung über diesen Gegenstand publicirte.

Da nun aber das Chinidin doch nicht an die Luft gesetzt werden konnte, so macht Herr Kerner von der schon von Winckler ${ }^{1}$ geübten Substitutionslehre Gebrauch und substituirt daher das gewöhnliche oder $\alpha$-Chinidin an Stelle der einen sogenannten Cinchonidinmodification, welche Stelle inzwischen vacant wurde. Dann will er uns glauben machen, dass er das fragliche Alkaloïd, dessen Sulfat dem Chininsulfat ähnlich krystallisirt, damals unter jener Modification verstanden hätte.

Herr Kerner wird sich erinnern, dass er die fragliche Modification nicht aus eignem Fabrikat, sondern aus einem aus Frankreich stammenden Sulfate de Quinidine darstellen konnte. Für den Kreis seiner Thätigkeit war mithin dieses Präparat etwas neues.

Nun wissen wir aber, dass in Frankreich damals zur Darstellung von Quinidine die Quinquina rouge de Mutis Verwendung fand, welche dort eigens den Namen Quinquina à Quinidine erbielt. Die Rinde enthält aber neben Cinchonin und etwas Cinchonidin (Winckler's Chinidin) namhafte Mengen Homocinchonidin, dessen Sulfat bekanntlich nicht in losen Krystallen in den Handel gelangt, sondern entweder in Form von Stücken oder als Pulver. Daher kann die $\beta$-Modification nicht wohl etwas anderes gewesen sein als die höchstens mangelhaft untersuchte $\alpha$-Modification seines Cinchonidins.

Uebrigens stelle ich durchaus nicht in Abrede, dass sein Cinchonidin und zwar die sogenannte $\alpha$ - wie $\beta$-Modification eine gewisse Menge von gewöhnlichem Chinidin enthielt, insbesondere als Herr Kerner jetzt, nach Verlauf von 18 Jahren, nach seinen eignen Angaben, diese beiden Alkaloïde noch nicht von einander zu unterscheiden und dem entsprechend doch auch nicht zu trennen weiss.

Es ist dies ein bedenkliches Zeichen für ihn; denn wenn er sich als Chinologe gerirt und sich dann anmaasst, über gewisse Chinaalkaloïde zu sprechen, so setzt das voraus, dass er diese Alkaloïde auch kenne. Diese Grundbedingung scheint aber Herrn Kerner Nebensache zu sein. So publicirte derselbe 1869, das

1) Annalen der Chemie und Pharmacie, 33, 308. 
Cinchonidin drehe die Ebene des polarisirten Lichtes nach rechts. Später, nachdem ich diese Angabe beanstandet hatte, schrieb mir Herr Kerner, dass dieses rechtsdrehende Cinchonidin nichts anderes als reines Cinchonin gewesen sei. Andererseits behauptete Herr Kerner für ein Chininsulfat, das ich zu untersuchen Gelegenheit hatte, den Gehalt von Cinchonin, während dieses vermeintliche Cinchonin Conchinin war n. s.w.

Unter diesen Umständen betrachtete es daher Herr Kerner fuir zeitgemäss, dass in dem sogenannten chinologischen Congress über den fraglichen Gegenstand gesprochen wurde. Das betreffende Protokoll besagt darüber wie folgt:

M. de Vrij entretient ensuite le Congrès de la confusion régrettable, qui c'est introduite dans la nomenclature des alcaloïdes du Quinquina. Il rapelle, que $M$. Pasteur a le premier defini nettement sous le nom de $Q$ uinidine un alcaloide isomère de la Quinine et sous celui de Cinchonidine un isomère de la Cinchonine. Depuis lors d'autres chimistes ont distingué ces memes corps sous des noms differents. M. Hesse en particulier a proposé pour un produit qui se confond avec la Quinidine, le nom de Conchinine. Il est évident, qu'on risque de ne plus s'entendre il faut en revenir aux definitions de M. Pasteur.

M. Schaer constate l'adhésion à cette opinion du plus grand nombre des pharmacologistes; il rapelle que dans la rédaction de la Pharmacopée helvètique il a suivi cette nomenclature.

M. Kerner est également d'avis que les observations de M. de Vrij doivent être prises en grande consideration et la réunion, à l'unanimité, émet le vocu que d'orénavant tous les chimistes acceptent le noms de $Q u i n i d i n e$ et de Cinchonidine dans le sens que leur a attribué M. Pasteur en 1853.

Von anderer Seite wurde in jenem Congress bezüglich dieser Sache Stillschweigen beobachtet. Hieraus schliesst aber Herr Kerner, dass ausser ihm Niemand zugegen war, der unter dem Namen Chinidin etwas anderes als die fragliche einzige Chininisomerie verstand. Gloichwohl schrieb mir Herr Kerner wenige Jahre vorher in Betreff des Begriffes Conchinin: „Gegen Ihren Namenswechsel babe ich, wie früher bemerkt, nichts einzuwenden, aber nicht, weil in Conchinin die Buchstaben des Cinchonins versetzt sind, sondern weil ich das $\mathrm{C}$ onchinin als Classenbindungswort zum Chinin hin für sehr geeignet halte." 
Dass de Vrij keine Gelegenheit versäumt, die sich inm darbietet, um gegen den Namen "Conchinin “ zu protestiren, ist eine altbekannte Sache. Als Gründe, die für die Bezeichnung Chinidin für das fragliche Alkaloïd sprechen sollen, werden der Reibe nach von ihm angefuihrt, dass Henry und Delondre das betreffende Alkaloïd entdeckt und unter dem Namen Chinidin beschrieben hätten, dass Pasteur es unter diesem Namen beschrieb und Gerhardt und Wöhler es später in ihren Lehrbüchern der organischen Chemie unter diesem Namen aufführten. Zuletzt machte de Vrij geltend, dass auch Howard es in seiner Quinology Chinidin nenne. In dem sogenannten chinologischen Congress greift derselbe, nach dem Protocoll, nur auf die betreffende Definition Pasteur's zurück. Nun aber publicirte schon 1852 Leers eine Abhandlung über Chinidin, in welchem derselbe klar und deutlich angiebt, was unter diesem Namen zu verstehen sei. Dies wird auch von Pasteur 1853 anerkannt. Aber Pasteur's käufliches Chinidin, das mir im Original vorliegt, enthielt als Beimengung 41 Proc. verwitternde Krystalle eines Alkaloïds, das mit dem von van Heyningen untersuchten $\beta$-Chinin übereinstimmt.

Es würde sich dann wohl gehört haben, dass Pasteur das vermeintliche eine ${ }^{1}$ Alkaloïd, welches den nicht verwitternden Antheil des fraglichen Chinidins ausmacht, wenigstens so weit dasselbe mit Leers Chinidin wirklich übereinstimmte, den Namen Chinidin belassen hätte, wozu ganz besonders die Thatsache hindrängte, dass das im Handel befindliche Chinidin vorzugsweise aus Leers Alkaloïd bestand, was Pasteur selbst anerkennt; den anderen offenbar ganz zufälligen Bestandtheil dagegen $\beta$-Chinin genannt hätte. Hierbei würde wenigstens dem historischen Rechte Genüge geleistet worden sein.

Pasteur vermuthete allerdings, dass das $\beta$-Chinin von van Heyningen nicht rein gewesen sei, ohne indess die Gründe anzugeben, die ihm zu dieser Vermuthung Veranlassung gaben. Dagegen habe ich 1868 hervorgehoben, dass das $\beta$-Chininsulfat van Heyningen's die Zusammensetzung des Chinidin-(Cinchonidin -) sulfat

1) In Wirklichkeit bestehen diese nicht verwitternde Krystalle aus zwei Alkaloïden: Cinchonidin und Homocinchonidin. Es ist das kein „Einfall“ von mir, wie Herr Kerner meint, sondern eine durch das Experiment ermittelte Thatsache. 
habe, woraus ich schloss, dass von van Heyningen's $\beta$-Chinin nicht frei von Leers' Chinidin gewesen sei. Wenn nun Herr Kerner, der sich tadelnd gegen diese Behauptung ausspricht, nicht ein Salz des fraglichen Alkaloïds darstellt, das thatsächlich 12,84 Proc. Krystallwasser enthält, so wird diese Behauptung in Kraft bleiben müвsen.

Zwar wird von de Vrij 1855 angeführt, dass er eine von van Heyningen erhaltene Probe $\beta$-Chinin identisch mit Pasteur's Chinidin gefunden habe, allein diese Angabe würde doch nur beweisen, dass van Heyningen neben seinem cinchonidinhaltigen Präparate auch noch reines Alkaloüd führte. Thatsächlich stimmen auch die Angaben van Heyningen's über das Chlorhydrat und Chloroplatinat mit den bezüglichen Eigenschaften des Conchininchlorhydrats und Conchininchloroplatinats recht gut überein, während bei den betreffenden Angaben über das neutrale Sulfat und Acetat das Cinchonidin za Tage tritt.

Pasteur nannte nun dieses Alkaloïd, für welches der Name $\beta$-Chinin unzweifelhaft historisch begründet ist, Chinidin, weil er glaubte, es liege in demselben des Chinidin von Henry und Delondre vor. Es wurde zwar sofort von Bouchardat und Boudet gezeigt, dass jenes Chinidin die Ebene des polarisirten Lichtes nach links ablenkte; allein die Autorität Pasteur's siegte über das unerbittliche Resultat des Experimentes und so kam es, dass die betreffenden Behauptungen Pasteur's bei Gerhardt und Wöhler Gehör fanden, welche diesen Irrthum dann durch ihre Lehrbücher weiter verbreiteten. Auch Delondre zögerte nicht, die ihm von Pasteur zugesprochene Entdeckung sofort anzunehmen, trotzdem dass Bouchardat und Boudet die Unvorsichtigkeit begangen hatten, Pasteur zu widersprechen. De Vrij, welcher später ein intimer Freund Delondre's wurde, gerirte sich damit zugleich als ein warmer Vertheidiger für diese schöne Entdeckung, welche aber von Delondre (in Gemeinschaft mit 0 . Henry) niemals gemacht wurde.

Delondre giebt in seinem mit Bouchardat herausgegebenen Werke über Chinarinden ganz genau an, welche Rinde das von ihm und Henry entdeckte Chinidin enthalten soll. Später habe ich darauf aufmerksam gemacht, dass die fragliche Rinde kein Conchinin (Pasteur's Chinidin) enthalte, sondern Cinchonidin beziehungsweise Homocinchonidin und dass daher Henry und Delondre's Substanz im wesentlichen mit diesem Alkaloüde übereinkomme, vielleicht 
auch noch eine rechtsdrehende Base enthalten habe, welch' letztere eventuell Cinchonin sein konnte. Bouchardat und Boudet fanden für das fragliche Chinidin, das in saurem $W$ asser gelöst war, ein Drehungsvermögen von $(\alpha) \mathbf{r}=-90,92^{\circ}$. Würde in diesem Präparate Pasteur's Chinidin vorgelegen sein, so würden sie mindestens $+330^{\circ}$ gefunden haben. Darauf hin hat Oudemans angenommen, dass jenes Chinidin wahrscheinlich ein Gemisch von Cinchonidin und Conchinin gewesen sei.

Die bezügliche Frage nahm 1877 dadurch eine andere Wendung, als Bouchardat, wahrscheinlich auf Anrathen von de Vrij, das originale Chinidin von Henry und Delondre eskamotirte und dafür ein von Delondre dargestelltes käufliches Chinidin erscheinen liess. Ingleichen wurde ausgefiihrt, dass die Quinquina rouge de Mutis gar nicht die Rinde wäre, aus welcher ursprünglich das fragliche Chinidin dargestellt worden sei. ${ }^{1}$

De Vrij theilte mir dann, als ich mit ibm in Cannstatt (1877) zusammentraf, mit, dass in der Bezeichnung der fraglichen Rinde von Seite Delondre's ein Irrthum stattgefunden habe und nach Delondre's Aussage die Quinquina jaune de Mutis die Rinde sei, aus welcher 1833 das in Rede stehende Alkaloïd dargestellt wurde. Allein von dieser Rinde, die auch seinem (Delondre's) Werke zur Unterlage diente, sei nichts mehr vorhanden, ingleichen von dem fraglichen Alkaloïde selbst.

In Betreff des letztern Punktes hat früher schon Flückiger ${ }^{2}$ die Bestätigung beigebracht. Schon 1873 sprach de Vrij, als er die weite Reise rom Haag nach Stuttgart unternahm, um mich von der Entdeckung des fraglichen Alkalọids zu überzeugen, die Henry und Delondre gemacht haben sollten, dahin aus, dass von jenem originalen Alkaloïd nichts mehr vorhanden sei. Um somehr überrascht mich jetzt die Mittheilung des Herrn Kerner, dass de Vrij in dem Besitze von diesem Präparate ist, welches derselbe sogar 1855 von Delondre selbst erhalten hat. Mit Recht darf ich

1) Henry und Delondre haben 1834 in einer Anmerkung zu ihrer Abhandlung über Chinidin angeführt, dass dasselbe mit Weinstein ein Doppelsalz bilde. Diese Angabe beruht entschieden auf einem Irrthum. Nähere Angaben über die zu nehmenden Mengen von Cremor Tartari und Alkaloïd fehlen zudem, so dass die von Herrn Kerner beigebrachten Zahlen 188 und 324 beziehungeweise 308 überflüssig erscheinen.

2) Dieses Archiv 210, 395. 
daher fragen: Warnm liess de Vrij 1873 gerade das wichtigste Beweismaterial zu Hause? Sollte wohl dieses plötzlich anfgetauchte Präparat inzwischen entstanden sein?

Wie dem auch sein mag, so erfahren wir jetzt durch Herrn Kerner, dass das Chinidin von Henry und Delondre nicht ein besonderes Alkaloïd, sondern ein Gemisch war. Es nimmt sich diese Thatsache eigenthümlich aus zu den fortgesetzten Behauptungen de Vrij's, nach welchen Pasteur's Chinidin ganz bestimmt von Henry und Delondre entdeckt worden sei.

Da Delondre die Rinden näher bezeichnete, welche das fragliche Chinidin enthalten, so habe ich die betreffenden Rinden 1 genau untersucht, in der Erwartang, selbst Aufschluss über die zweifelhafte Base zu erhalten. Diese Untersuchung ergab für 100 Thle. Quinquina

jaune de Mutis rouge vîf rouge pâle

\begin{tabular}{|c|c|c|c|}
\hline Chinin & 0,96 & 1,33 & 1,26 \\
\hline Chinidin & 0,17 & 0,51 & 1,88 \\
\hline Conchinin . & Spur & 0,00 & Spur \\
\hline Cinchonin & 0,27 & 0,55 & 0,59 \\
\hline \multirow{2}{*}{ Amorphe Basen } & 0,47 & 1,66 & 1,92 \\
\hline & 1,87 & 4,05 & 5,65 . \\
\hline
\end{tabular}

Dieses Chinidin ist nun nichts anderes als das von Leers untersuchte Alkaloïd. Würden wir versuchen, aus der Quinquina jaune de Mutis nach der frühern Art Chininsulfat darzustellen, so würde dasselbe unzweifelhaft chinidinhaltig werden. Noch mehr würde aber die letztere Erscheinung bei Anwendung der beiden andern Rinden stattfinden. Ein nicht unerheblicher Theil Chinidin würde übrigens in die Mutterlauge übergehen, dazu die ganze Menge Conchinin, so dass es dunn möglich wäre, dass das aus dieser Lauge dargestellte Chinidin etwas Conchinin enthalten könnte, wenigstens bei Anwendung der Quinquina jaune ocre de Mutis (so nannte Delondre auch die fragliche Rinde). In allen Fällen müsste aber das erhaltene Chininsulfat conchininfrei sein. Wenn nun einestheils Henry anfübrt, dass zur Darstellung des Chinidins Chininsulfat angewendet werden kann und derselbe

1) Diese Rinden sind der Delondre'schen Sammlung entnommen, welche damal (1877) im Besitze Wiggers war und jetzt an die Göttinger Universität übergegangen ist. 
anderntheils bei einem Gehalt der Rinde von 0,21 Proc. Conchinin, welche Mengen neben Spuren von Chinidin die Quinquina de pitago enthielt, kein Chinidin erhalten konnte, so folgt daraus mit Bezug auf die obigen Analysen, dass jenes Chinidin eben nichts anderes als Leers Chinidin sein konnte.

Uebrigens habe ich wiederholt Proben von Chininsulfat aus früher Zeit untersucht und französische Fabrikate namentlich chinidinhaltig gefunden, welches Resultat zu der bezüglichen Angabe Henry's stimmen würde. Niemals gelang es mir aber, in solchem Sulfat Conchinin nachzuweisen, obwohl solche Methoden angewendet wurden, welche selbst minutiöse Mengen Conchinin erkennen liessen.

Was die Qualität von Pasteur's Chinidin betrifft, so muss man darüber trotz der gegentheiligen Behauptung de Vrij's heute in Zweifel sein. Pasteur findet nämlich das Drehungsvermögen des fraglichen Alkaloïds in der Auflösung in absolutem Alkohol zu $(\alpha) \mathrm{j}=+250,75^{\circ}$, Oudemans dagegen $(\alpha) \mathrm{D}=+255,4^{\circ}$. Nach Herrn Kerner ist natïrlich eine solche Uebereinstimmung ein schlagender Beweis dafür, dass der Begriff „Chinidin" von Pasteur genau präcisirt wurde. Indess muss daran erinnert werden, dass das Zeichen ,j “ eine ganz andere Bedeutung als das Zeichen „D“ hat. Der Werth $(\alpha)$ j lässt sich nicht direct aus $(\alpha) D$ ableiten, weil die Dispersion des Lichtes bei den verschiedensten Substanzen verschieden ist. Wahrscheinlich beträgt $(\alpha) \mathrm{j}$ für Conchinin unter den von Pasteur eingehaltenen Verhältnissen $+280-290^{\circ}$. In jedem Falle trifft daher Herrn Kerner's bezügliche Behauptung nicht zu.

Ich hatte früher irrthümlich angegeben, dass sich Pasteur's Beobachtung auf rothes Licht bezieht. Zu diesem Irrthum kam ich lediglich durch die betreffenden Angaben im (alten) Handwörterbuch für Chemie und in Husemunn's Pflanzenstoffe, in welchen Werken man $(\alpha) \mathrm{r}$ anstatt $(\alpha) \mathrm{j}$ angegeben findet. Später habe ich diesen Irrthum bemerkt und auch berichtigt. ${ }^{1}$ Es würde daher Herr Kerner, wenn er ein Freund der Wabrheit wäre, wohl daran gethan haben, von dieser Berichtigung Notiz zu nehmen, anstatt in der ihm eignen Art diesen Irrthum auszubeuten.

Die optischen Unterschiede, welche Pasteur für die verwitternden und nicht verwitternden Krystalle seines käuflichen Chinidins

1) Liebig's Annalen, 176, 317. 
kennen lehrte, bestimmten schon de Vrij frühzeitig, das Polariskop für alle ihm unter die Hände gelangenden Präparate in Anwendung zu bringen. Es gelang ihm schliesslich, wie Derselbe behauptete, nicht nur mit diesem Apparate die einzelnen Chinaalkaloïde von einander $\mathrm{zu}$ unterscheiden und sie qualitativ $\mathrm{zu}$ bestimmen, sondern auch die Rinde der Cinchona succirubra zu erkennen. Und das alles zu einer Zeit, wo noch nicht bekannt war, dass das betreffende optische Verhalten durch die Concentration der Lösung, durch die Qualität der chemisch indifferenten und chemisch activen Lösungsmittel, durch die Art der chemischen Bindung und endlich durch die Temperatur bedingt ist. An einem andern Orte habe ich angedeutet, dass alle diese bezüglichen Versuche de Vrij's werthlos sind, weil eben die erwähnten Factoren dabei mehr oder weniger unberücksichtigt blieben. Auch Herr Kerner konnte sich, wie mir derselben vor wenigen Jahren schrieb, nicht für jene optischen Untersuchungen de Vrij's besonders erwärmen.

Nach der Lobhudelei zu urtheilen, welche Herr Kerner jetzt de Vrij zukommen lässt, muss man annehmen, dass zwischen Beiden etwas vorgegangen ist, welches das Eis schmelzen machte.

Herr Kerner kommt deshalb auch auf die genaue Vorschrift zurück, welche de Vrij gab, um Conchinin nachzuweisen. Bekanntlich bildet dieses Alkaloüd mit Jodwasserstoff ein in Wasser und Alkohol schwer lösliches Salz, in Folge dessen diese Säure als Reagens auf Conchinin empfohlen wurde. Herr Kerner deutet aber diese Angabe so, als hätte de Vrij damit eine Conchininprobe bekannt gegeben. Eine Reaction ist aber noch keine Probe, sondern letztere entsteht erst, wenn die fragliche Reaction in passender Weise angewendet wird. Das wusste auch de Vrij recht wohl, indem er 1878 nachträglich eine Conchininprobe bekannt machte. Allerdings ist diese Probe nichts weiter als die Copie meiner Probe, die ich 1875 zur Prüfung des Conchininsulfats in Liebig's Annalen 176, 322 publicirte. Wenn ich nun später auf diese Copie aufmerksam machte und endlich meine Conchininprobe in gedrängter Form in diesem Archiv nachträglich bekannt machte, so kann ich daran nichts komisches finden. Dagegen kommt mir die betreffende Behauptung des Herrn Kerner sehr sonderbar vor.

Obgleich nun de Vrij Jodwasserstoff als ein vorzügliches Reagens auf Conchinin angab, so hat derselbe trotzdem bisweilen erhebliche Mengen Conchinin übersehen, wie ich früher schon her- 
vorgehoben habe. Als Beleg hierfür diente namentlich das Resultat einer Rinde. Auch die häufiger vorkommenden Alkaloïde kannte de Vrij 1869 ganz sicher zu wenig. Dass diese meine Behauptung, welche Herr Kerner nach seiner Art auslegte, ganz richtig ist, bewies ja später de Vrij in eklatanter Weise selbst, indem er z. B. Cinchonidin mit Natriumbitartrat zum Theil ausfällte und den beträchtlichen Rest dieses Alkaloïdes, welcher noch in Lösung sich befand, für ein neues Chinaalkaloïd ansprach.

Ich habe später de Vrij brieflich auf diesen Irrthum aufmerksam gemacht und ihn gebeten, er möchte die bezügliche Angabe über das vermeintlich neue Alkaloïd zurücknehmen. Da nun aber die Zurücknahme nicht erfolgte - sie müsste mir denn entgangen sein -, so habe ich denn selbst auf die Nichtexistenz dieses angeblich neuen, nach links polarisirenden Alkaloïds hingewiesen. Herr Kerner ist darüber anderer Ansicht, denn er sagt, es ergebe sich hieraus, wie ungenirt und ungerechtfertigt ich Andern zur Erhöhung des eigenen Reliefs Verdienste abspreche. Angesichts dieser Beschuldigung erlaube ich mir noch die Analyse von krystallisirbarem Chinin mitzutheilen, welches de Vrij aus javanischer Calisayarinde darstellte und mir 1873 gab. Dasselbe enthält in 100 Theilen

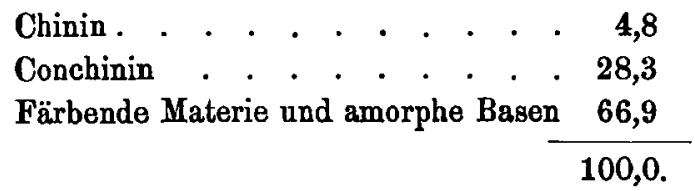

Dieses krystallisirbare Chinin enthält also nur wenige Procente wirkliches Chinin und trotz der genauen Fällungsmethode mit Jodwasserstoff fast ein Drittheil Conchinin als Rückhalt.

Rechne ich nun hierzu, dass de Vrij aus mir bekannten Gründen hätte wissen müssen, dass Pasteur's Cinchonidin ein Gemisch von Winckler's Chinidin und Koch's Cinchonidin sei, ferner dass Herr Kerner jetzt noch darauf wartet, bis ich ihm das Nähere betreffs der Trennung und der Eigenschaften der fraglichen beiden Alkaloïde mittheile, so verstehe ich nicht, wie diese Herren bei ihrer notorisch mangelhaften Kenntniss der Sache berechtigt sein können, die Nomenclatur der fraglichen Substanzen zu normiren. 